\title{
In silico secretome characterization of clinical Mycobacterium abscessus isolates provides insights into antigenic differences
}

Fernanda Cornejo-Granados ${ }^{1}$, Thomas A. Kohl ${ }^{2,3}$, Flor Vásquez Sotomayor ${ }^{4}$, Sönke Andres $^{4}$, Rogelio Hernández-Pando ${ }^{5}$, Juan Manuel Hurtado-Ramírez ${ }^{1}$, Christian Utpatel $^{2,3}$, Stefan Niemann ${ }^{2,3}$, Florian P. Maurer ${ }^{4,6, \# *}$ and Adrian Ochoa-Leyva a, ${ }^{1, * *}$

${ }^{1}$ Departamento de Microbiología Molecular, Instituto de Biotecnología, Universidad Nacional Autonoma de México, Cuernavaca, Morelos, México.

2 Molecular and Experimental Mycobacteriology, Research Center Borstel, Borstel, Germany

${ }^{3}$ German Center for Infection Research (DZIF), Partner site Hamburg-Lübeck-Borstel, Borstel, Germany

${ }^{4}$ National and WHO Supranational Reference Center for Mycobacteria, Research Center Borstel, Leibniz Lung Center, Borstel, Germany

${ }^{5}$ Experimental Pathology Section, National Institute of Medical Sciences and Nutrition Salvador Zubirán, Mexico City, Mexico.

${ }^{6}$ Institute of Medical Microbiology, Virology and Hospital Hygiene, University Medical Center Hamburg-Eppendorf, Hamburg, Germany

\# equal contribution

\section{Correspondance:}

*co-corresponding authors:

Adrian Ochoa-Leyva: aochoa@ibt.unam.mx

Florian P. Maurer: fmaurer@fz-borstel.de

Keywords: bioinformatics, antigenicity, M. abscessus subspecies, in silico analysis, vaccinology. 


\begin{abstract}
Mycobacterium abscessus (MAB) is a widely disseminated pathogenic non-tuberculous mycobacterium (NTM). Like with M. tuberculosis complex (MTBC), excreted / secreted (ES) proteins play an essential role for its virulence and survival inside the host. ES proteins contain highly immunogenic proteins, which are of interest for novel diagnostic assays and vaccines. Here, we used a robust bioinformatics pipeline to predict the secretome of the M. abscessus ATCC 19977 reference strain and fifteen clinical isolates belonging to all three MAB subspecies, $M$. abscessus subsp. abscessus, M. abscessus subsp. bolletii, and M. abscessus subsp. massiliense. We found that $\sim 18 \%$ of the proteins encoded in the MAB genomes were predicted as secreted and that the three MAB subspecies shared > $85 \%$ of the predicted secretomes. MAB isolates with a rough (R) colony morphotype showed larger predicted secretomes than isolates with a smooth (S) morphotype. Additionally, proteins exclusive to the secretomes of MAB R variants had higher antigenic densities than those exclusive to $\mathrm{S}$ variants, independently of the subspecies. For all investigated isolates, ES proteins had a significantly higher antigenic density than non-ES proteins. We identified 337 MAB ES proteins with homologues in previously investigated M. tuberculosis secretomes. Among these, 222 have previous experimental support of secretion, and some proteins showed homology with protein drug targets reported in the DrugBank database. The predicted MAB secretomes showed a higher abundance of proteins related to quorum-sensing and Mce domains as compared to MTBC indicating the importance of these pathways for MAB pathogenicity and virulence. Comparison of the predicted secretome of M. abscessus ATCC 19977 with the list of essential genes revealed that 99 secreted proteins corresponded to essential proteins required for in vitro growth. All predicted secretomes were deposited in the Secret-AAR web-server (http://microbiomics.ibt.unam.mx/tools/aar/index.php).
\end{abstract}




\section{Introduction}

Non-tuberculous mycobacteria (NTM) are widely disseminated, mostly saprophytic and partly opportunistic bacteria. The prevalence of NTM in clinical specimens has increased globally, and in some industrialized countries, infections caused by NTM are becoming more common than tuberculosis (TB). Infections caused by M. abscessus (MAB) are particularly challenging to manage due to the extensive innate resistance of MAB against a wide spectrum of clinically available antimicrobials (Nessar et al., 2012). MAB causes mostly pulmonary and occasionally extrapulmonary infections that can affect all organs in the human body (Lee et al., 2015). Current treatments for MAB induced pulmonary disease are long, associated with severe side effects and a cure rate below $50 \%$ (Chen et al., 2019; Jarand et al., 2011; Sanguinetti et al., 2001). MAB is comprised of three subspecies, M. abscessus subsp. abscessus, M. abscessus subsp. bolletii and M. abscessus subsp. massiliense, hereafter referred to as $\mathrm{MAB}_{\mathrm{A}}, \mathrm{MAB}_{\mathrm{B}}$, and $\mathrm{MAB}_{\mathrm{M}}$, respectively (Tortoli et al., 2016). MAB isolates can show smooth (S) and rough (R) colony morphotypes, a trait that relies on the presence $(\mathrm{S})$ or absence $(\mathrm{R})$ of surface- associated glycopeptidolipids (GPLs) and that correlates with the virulence of the strain (Abeles and Pride, 2014; Howard et al., 2006; Ripoll et al., 2007) (Gutiérrez et al., 2018)). Transitioning from high-GPL to low-GPL production is observed in sequential MAB isolates obtained from patients with chronic underlying pulmonary disease. In these patients, S-to-R conversion is thought to present a selective advantage as the aggregative properties of MAB R variants strongly affect intracellular survival. In addition, a propensity to grow as extracellular cords allows these low-GPL producing bacilli to escape innate immune defenses (Gutiérrez et al., 2018).

The complete set of proteins excreted / secreted (ES) by a bacterial cell is referred to as its secretome. The secretome is involved in critical biological processes such as cell adhesion, migration, cell-to-cell communication and signal transduction (Tjalsma et al., 2004) ES proteins are considered an important source of molecules for serological diagnosis. Also, secreted proteins can be highly antigenic due to their immediate availability to the host immune system and are thus of interest in vaccinology (Daugelat et al., 1992; Zheng et al., 2013). So far, there have been few efforts to experimentally determine the secretome of $\mathrm{MAB}$, and in particular, the secretomes of clinical MAB isolates (Gupta et al., 2009; Laencina et al., 2018; Shin et al., 2010; Yadav and Gupta, 2012). Nowadays, sequencing and bioinformatics strategies can be explored for the systematized prediction of ES proteins from bacterial genomes (Cornejo-Granados et al., 2017; Gomez et al., 2015). Recently, a robust bioinformatics pipeline for predicting and analyzing the complete in silico secretome of two clinical M. tuberculosis (MTB) genomes was published in 2017 by our group showing higher overall agreement with an experimental secretome compiled from literature than two previously reported secretomes for M. tuberculosis H37Rv (Cornejo-Granados et al., 2017).

To gain further insights into MAB ES proteins and their association with virulence and pathogenicity we sequenced and assembled the genomes of fifteen clinical MAB isolates belonging to all three subspecies including $\mathrm{S}$ and $\mathrm{R}$ morphotypes. We then adapted the bioinformatics strategy previously established for MTB to predict and analyze the complete set of ES proteins encoded in these isolates and in the M. abscessus ATCC 19977 type strain, and compared it with our previous findings for MTB (Cornejo-Granados et al., 2017). 


\section{Materials and methods}

\section{Ethics statement}

Ethical review and approval was not required for the study as all work was performed on bacterial isolates archived at the strain repository of the National Reference Center for Mycobacteria in Borstel, Germany, in accordance with local legislation and institutional requirements. In particular, no data allowing identification of the affected patients was shared or released and no human DNA was sequenced or analyzed.

\section{Clinical isolates}

We selected fifteen $\mathrm{MAB}$ clinical isolates comprising members of all MAB subspecies $\left(\mathrm{MAB}_{\mathrm{A}}, \mathrm{n}=7 ; \mathrm{MAB}_{\mathrm{B}}, \mathrm{n}=4 ; \mathrm{MAB}_{\mathrm{M}}, \mathrm{n}=4\right)$ and both $\mathrm{S}(\mathrm{n}=8)$ and $\mathrm{R}(\mathrm{n}=6)$ morphotypes (not determined, $\mathrm{n}=1$ ). The strains were isolated from different biological sources representing both pulmonary colonization / infection (sputum, $\mathrm{n}=10$ ) and extrapulmonary samples (skin, $\mathrm{n}=1$; soft tissue, $\mathrm{n}=1$; lymph nodes, $\mathrm{n}=2$; blood, $\mathrm{n}=1$ ) (Table 1and S1). For routine diagnostic purposes, species identification was performed using GenoType NTM-DR line probe assays (HAIN Lifescience, Nehren, Germany) and sequencing of the $16 \mathrm{~S}$ and $r p o B$ genes as described previously (Adekambi et al., 2003).

\section{Whole genome sequencing and genome assemblies}

Genomic DNA (gDNA) of the 15 MAB clinical isolates was extracted from solid cultures using a Centrimonium bromide chloroform DNA extraction protocol as previously described (De Almeida et al., 2013). DNA libraries were constructed with the Nextera XT kit from Illumina and sequenced on the Illumina MiSeq benchtop platform with a v3 chemistry paired -end run and a read lenght of 2x300 bp. We processed the resulting reads with Trimmomatic (Bolger et al., 2014), clipping the Illumina adapter sequences and trimming the reads with a sliding window of $20 \mathrm{bp}$ looking for quality $>30$ and discarding all reads shorter than $100 \mathrm{bp}$. Trimmed reads were used to construct de novo assemblies using SPADES (Nurk et al., 2013) with default parameters and the --careful option enabled. Then, each assembly was analyzed with RAST (Aziz et al., 2008) to obtain all the open reading frames (ORFs). Additionally, we predicted the ORFs from the deposited genome sequence of the M. abscessus ATCC 19977 type strain (GenBank CU458896.1) (Supplementary Table S1).

\section{Secretome prediction}

The complete set of predicted ORFs was independently analyzed for each genome using the bioinformatics pipeline previously reported by Cornejo-Granados et al., 2017 and summarized in Supplementary Fig. S1. Briefly, we used six different feature-based tools (SignalP, SecretomeP, LipoP, TatP, TMHMM and Phobius) (Bendtsen et al., 2005a; 2005b; Petersen et al., 2011; Sonnhammer et al., 1998) (Juncker et al., 2003; Käll et al., 2007) to identify ES proteins by the different secretion pathways and to remove the ones that had 
171

172

173

174

175

176

177

178

179

180

181

182

183

184

185

186

187

188

189

190

191

192

193

194

195

196

197

198

199

200

201

202

203

204

205

206

207

208

209

210

211

212

213

214

215

216

transmembrane domains (Supplementary Fig. S1). The proteins assigned as not-secreted (non-ES) were further classified into transmembrane proteins (TM) if they showed the presence of transmembrane domains with TMHMM 2.0 (Sonnhammer et al., 1998), and into intracellular proteins (incell) if they did not contain any transmembrane domains.

\section{Annotation and comparative analysis of secreted proteins}

To assign functional annotations to the proteins present in our genomes, we performed a BLASTP query of those proteins against the non-redundant (nr) complete database using Blast2GO (Conesa and Götz, 2008) with an E-value cut-off set at 1.0E-3. Furthermore, all proteins were associated with protein families through InterProScan (Zdobnov and Apweiler, 2001) and functionally mapped to Gene Ontology (GO) terms by setting the following parameters: E-value-hi-filter: 1.0E-3; Annotation cut-off: 55; GO weight: 5 and Hsp-Hit Coverage cut-off: 0 . Blast2GO was then used to identify over- and under-represented GO and Enzyme Commission (EC) numbers in the ES proteins by setting the significance filter p-value to $\leq 0.05$. Also, we used the KEGG Automatic Annotation Server (KAAS) database (Moriya et al., 2007) to assign the pathway annotation to the secreted proteins using the BBH (bidirectional best hit) method and the gene data set assigned to Mycobacterium.

To determine differences between the predicted secretomes in relation to MAB subspecies and morphotype, we established core secretomes by performing a bidirectional best-hit BLASTP search (E-value 1.0E-3) between the ES proteins of all genomes belonging to the respective subspecies and morphotypes. Then, we identified the shared and unique proteins for each comparison. Additionally, we determined the ES proteins shared between the MAB reference strain ATCC 19977 and M. tuberculosis H37Rv predicted and experimental secretomes (Cornejo-Granados et al., 2017). The resulting shared proteins were further investigated for sequence similarities against known drug targets available on the Drug Bank database (http://www.drugbank.ca/), setting the E-value to $1.0 \mathrm{E}-3$ and all other options to default. In Supplementary Table S2, we show all proteins that have similarity with an approved drug target, as well as the drugs that can affect said target.

Additionally, we analyzed the presence of the core secretomes in twenty $M$. abscessus genomes per subspecies downloaded from NCBI (Supplementary Table S3). To this end, each downloaded genome was analyzed with RAST to obtain all the open reading frames (ORFs). Next, we performed a BLASTP search (E-value 1.0E-3) of each core secretome against each genome of the corresponding subspecies, and all hit proteins were considered homologs.

\section{Calculation of the Abundance of Antigenic Regions}

The Abundance of Antigenic Regions (AAR) value is used to estimate the antigenic density of a protein by calculating the number of antigenic regions and normalizing it to the sequence length (Gomez et al., 2015). Of note, proteins with higher antigenic densities have lower AAR values. For this study, we calculated the AAR value for each protein in each data set using the Secret-AAR web-server (http://microbiomics.ibt.unam.mx/tools/aar/index.php) and reported the average unless stated otherwise (Cornejo-Granados et al., 2018). Then, we used a Mann-Whitney 
statistical test to establish any significant differences between the AAR values of the different protein data sets.

\section{Data availability}

221 The reference genomes analyzed for M. abscessus ATCC19977 and M. tuberculosis H37Rv were taken from NCBI, under GenBank IDs CU458896.1 and NC_000962.3, respectively. The Whole Genome Shotgun project has been deposited at NCBI, under BioProject PRJNA646278. All the predicted secretomes were deposited in the Secret-AAR web-server (http://microbiomics.ibt.unam.mx/tools/aar/index.php).

\section{Results}

\section{Genome assembly, secretome prediction and annotation}

We sequenced the genomes of fifteen pulmonary and extrapulmonary (skin, tissue, and lymph node, blood) MAB isolates obtained from patients in Germany comprising all three MAB subspecies (Table 1 and S1). For each genome, we obtained an average of 2,601,444 quality-filtered reads. After de novo assembly, we obtained from 38 to 78 contigs ( mean $=58$ contigs) with genome coverage of 217- to 368-fold (mean $=310$-fold) and with an average of 5,082 total proteins per genome (Supplementary Table S4).

We used a bioinformatics pipeline previously reported by our group (Cornejo-Granados et al., 2017) to predict the full secretome of all MAB clinical isolates and the widely used reference strain M. abscessus ATCC 19977 (GenBank CU458896.1) (Supplementary Fig. S1). We obtained an average of 939 ES proteins per genome, representing $\sim 18 \%$ of the total proteome (Table 1). The predicted secretome for the MAB reference strain consisted of 886 proteins. From these, all the proteins showed a BLASTP hit against the NR database, and only 494 (55.8\%) could be annotated with GO terms.

We analyzed the over-representation of GO terms in the secretome of M. abscessus ATCC 19977 as compared to the whole genome. The most significantly enriched GO-terms were: "lytic vacuole" ( $p=9.37 \mathrm{E}-04)$ and "fungal-type vacuole" $(p=0.004)$ in Cellular Component (Fig. 1A), "serine-type carboxypeptidase" $(p=1.83 \mathrm{E}-04)$, and "serine-type DAla-D-Ala carboxypeptidase" ( $p=1.83 \mathrm{E}-04)$ activities in Molecular Function (Fig. 1B) and "response to inorganic substance" ( $\mathrm{p}=5.68 \mathrm{E}-04)$ and "cellular response to oxygen radical" ( $\mathrm{p}=0.001$ ) in the Biological Process category (Fig. 1C). The KEGG pathway mapping of the ES proteins showed that 214 proteins $(24.2 \%)$ could be assigned to 100 different KEGG pathways (Table 2), with the ABC transporter pathway being the most abundant ( $\mathrm{n}=$ $13,1.47 \%)$. Additionally, serine-type D-Ala-D-Ala carboxypeptidases $(\mathrm{p}=1.83 \mathrm{E}-04)$ and peptidases $(\mathrm{p}=8.40 \mathrm{E}-04)$ were the most significantly abundant enzymes according to the Enzyme Commission (EC) Classes (Figure S2), while the Mce/MiaD and PknH-like extracellular domains were the most enriched protein domains (Table 3). Of note, comparably few sequences were assigned to the PE/PPE category $(n=3)$. Notably, after comparing the predicted secretome of $M$. abscessus ATCC 19977 with a list of essential genes published by (Laencina et al., 2018), we found that $99(11.17 \%)$ of the predicted ES proteins, corresponded to essential proteins required for in vitro growth.

\section{Comparison of $\mathrm{M}$. abscessus subspecies core secretomes}


We analyzed the differences between the predicted secretomes of the three MAB subspecies. To this end, we defined the core secretome of each subspecies as the set of proteins shared between all secretomes of isolates belonging to $\mathrm{MAB}_{\mathrm{A}}, \mathrm{MAB}_{\mathrm{B}}$, and $\mathrm{MAB}_{\mathrm{M}}$, respectively. The resulting core secretomes contained $735\left(\mathrm{MAB}_{\mathrm{A}}\right), 794\left(\mathrm{MAB}_{\mathrm{B}}\right)$, and 813 $\left(\mathrm{MAB}_{\mathrm{M}}\right)$ proteins (Fig $\left.2 \mathrm{~A}\right)$.

Given that our study considered a limited number of de novo assembled genomes, we additionally compared the predicted core secretomes to sixty additional MAB genomes available in NCBI (Supplementary Table S3). We found that an average of 99.78\%, 99.12\%, and $98.59 \%$ of our core secretomes was also present in the investigated additional $\mathrm{MAB}_{\mathrm{A}}$, $M A B_{B}$, and $M_{A B}$ genomes, respectively, further corroborating the validity of the predicted subspecies core secretomes for other MAB isolates.

We then determined the respective AAR values to estimate antigenic densities for the protein sets in each core secretome. The average AAR values from most to least antigenic was: 40.24 for $\mathrm{MAB}_{\mathrm{A}}, 40.75$ for $\mathrm{MAB}_{\mathrm{B}}$, and 41.38 for $\mathrm{MAB}_{\mathrm{M}}$ with no statistically significant difference between these values.

Next, we identified the ES proteins shared between the $\mathrm{MAB}_{\mathrm{A}}, \mathrm{MAB}_{\mathrm{B}}$, and $\mathrm{MAB}_{\mathrm{M}}$ core secretomes. We found that 704 proteins $(86.5 \%)$ were shared among $\mathrm{MAB}_{\mathrm{A}}, \mathrm{MAB}_{\mathrm{B}}$, and $\mathrm{MAB}_{M}$ with an AAR value of 41.17 (Fig. 2B). The AAR values for the protein sets exclusively found in the $\mathrm{MAB}_{\mathrm{A}}, \mathrm{MAB}_{\mathrm{B}}$, or $\mathrm{MAB}_{\mathrm{M}}$ secretome were 33.58, 41.22, and 43.13, respectively, with the $\mathrm{MAB}_{\mathrm{A}}$ dataset showing a significantly lower AAR value indicating higher antigenicity than the others $(p<0.1$; Fig. $2 B)$.

\section{Differences in core secretomes between $R$ and $S$ morphotypes}

As MAB isolates with $\mathrm{R}$ and $\mathrm{S}$ morphotypes show differences in virulence and pathogenicity, we compared the predicted core secretomes of $\mathrm{R}$ and $\mathrm{S}$ isolates (Fig. 3). We observed that the core secretomes of $\mathrm{R}$ variants were larger (840, 924 and 845 proteins for $\mathrm{MAB}_{\mathrm{A}}, \mathrm{MAB}_{\mathrm{M}}$, and $\mathrm{MAB}_{\mathrm{B}}$ ) than those of the investigated $\mathrm{S}$ variants $(764,872$ and 833 proteins, respectively) with no significant differences in antigenic densities as per mean AAR values (Fig. 3). Intra-subspecies comparison of S and R secretomes revealed that 96.4 $\%, 90.7 \%$ and $95 \%$ of the identified ES proteins were found in both $\mathrm{R}$ and $\mathrm{S}$ morphotypes for $\mathrm{MAB}_{\mathrm{A}}, \mathrm{MAB}_{\mathrm{M}}$ and $\mathrm{MAB}_{\mathrm{B}}$ respectively. The number of unique proteins was larger in the core secretome of the $R$ morphotypes $\left(n=93,109\right.$, and 48 for $\mathrm{MAB}_{\mathrm{A}}, \mathrm{MAB}_{\mathrm{M}}$, and $\left.\mathrm{MAB}_{\mathrm{B}}\right)$ as compared to the $\mathrm{S}$ morphotypes $(\mathrm{n}=9,76$, and 35, respectively; Fig.3). Interestingly, antigenic densities for the unique ES proteins of the $\mathrm{R}$ morphotypes were higher $\left(\mathrm{AAR}=40.84,36.71\right.$, and 35.59 for $\mathrm{MAB}_{\mathrm{A}}, \mathrm{MAB}_{\mathrm{M}}$, and $\left.\mathrm{MAB}_{\mathrm{B}}\right)$ than for the proteins exclusive to the $S$ morphotypes irrespective of the subspecies (AAR $=45.43$, 37.72, and 42.14; Fig. 3). To assess if the AAR values of these specific protein sets were different from same-sized protein sets randomly chosen from the respective core secretomes, we created 1000 random sets of 109, 93, 76, 48, 35 and 9 proteins and calculated the AAR value for each set. Then, we determined an empirical p-value based on the number of random protein sets that equaled or exceeded the AAR value for each protein dataset as was previously suggested by Cornejo-Granados et al., 2017. We found that the ES proteins exclusive to the $R$ morphotypes of $M_{M} B_{M}$ and $M A B_{B}$ had significantly ( $p$ $<0.05)$ higher antigenic densities than randomly constructed protein sets (Supplementary Table S5). 
Finally, we determined the MAB core secretomes by sample origin (pulmonary,

\section{Antigenicity of ES and non-ES proteins}

It has previously been reported for different microorganisms including MTB that ES proteins tend to be more antigenic than non-ES proteins (Cornejo-Granados et al., 2017; Gomez et al., 2015; Wang et al., 2015). We thus tested if this was also true for the investigated MAB isolates. First, we found that the antigenic densities as indicated by mean AAR values were very similar among all isolates irrespective of subspecies or morphotype within the same cell compartment, i.e. for ES, non-ES, intracellular (incell) and transmembrane (TM) proteins (Fig. 4). Second, we found that antigenic densities were significantly higher in ES proteins as compared to non-ES proteins in all isolates (AAR = 40.57 and 43.60, respectively; p-value < 0.0001) (Fig. 4). However, within the non-ES category, incell proteins showed even higher antigenic densities $(\mathrm{AAR}=39.04)$ than the predicted ES proteins $(\mathrm{p}<0.0001)$ while the lowest overall antigenic densities were observed for the TM category $(\mathrm{AAR}=59.23$; $\mathrm{p}<0.0001)$.

\section{Comparison of M. abscessus and M. tuberculosis secretomes}

Lastly, we compared the predicted secretome of M. abscessus ATCC 19977 against the previously reported secretome of M. tuberculosis H37Rv (Cornejo-Granados et al., 2017). We observed that the $M$. abscessus secretome was predicted to be almost equally antigenic ( $\mathrm{AAR}=40.78$ ) than the $M$. tuberculosis secretome (AAR=40.63) (Fig. 5). We found 337 MAB ES proteins (38.04\%) with homology to proteins in the predicted MTB secretome (Fig. 5). In addition, 222 of these proteins had sequence homology with proteins experimentally reported as secreted in MTB (comparable experimental secretome data for MAB was not available to us) (Cornejo-Granados et al., 2017) (Supplementary Table S6). Furthermore, we determined the average AAR value of the 680 ES proteins shared among the fifteen $\mathrm{MAB}$ isolates $(\mathrm{AAR}=41.53)$. This value means that antigenic density was lower than for the predicted secretome of M. tuberculosis H37Rv (AAR $=40.63$ ) and two clinical M. tuberculosis isolates belonging to the Beijing lineage (isolate C3 AAR $=37.51$ and isolate C4 AAR = 37.54,) (Table 4) (Cornejo-Granados et al., 2017). Finally, we identified 17 ES proteins with homologues in both MAB and M. tuberculosis, which are listed as targets for various FDA approved drugs (Supplementary Table S2).

\section{Discussion}

This is the first study that proposes a method for prediction of MAB secretomes based on fifteen clinical MAB isolates and the M. abscessus ATCC 19977 reference strain. Our results show that an average of $18 \%$ (939 proteins) of the total proteins encoded in the MAB core genome carry sequence patterns indicative of secretion. Notably, this percentage 
is $6 \%$ larger than the proportion previously reported for several MTB isolates $(\sim 12 \%)$ (Cornejo-Granados et al., 2017). Nearly 200 species of mycobacteria have been identified with diverse lifestyles and a high degree of morphological, biochemical, and physiological diversity and a comparative genome analysis suggests that only a relatively small number of genes (1080) are shared between several Mycobacterium species (Malhotra et al., 2017; Tortoli et al., 2017). Moreover, loss of ancestral genes is a well described phenomenon in slowly growing mycobacteria such as MTB and, in particular, M. leprae (Bachmann et al., 2019). In contrast, rapidly growing NTM such as MAB are considered to represent a more ancient evolutionary state, with larger genomes than those of MTB (Bachmann et al., 2019; Malhotra et al., 2017). Thus, it is not surprising that we found a larger number of ES proteins in MAB than MTB. Furthermore, the increased abundance of ES proteins in MAB as compared to MTB could be related to the ability of MAB to cause a different spectrum of disease and to adapt to different environmental settings requiring frequent interaction with a wide variety of host cells and organisms competing for the same ecological niche, likely involving cross species exchange of genetic information, for example by plasmid transfer (Ripoll et al., 2009; Ryan and Byrd, 2018; Waman et al., 2019). A similar hypothesis has been suggested for fungal secretomes (O'Toole et al., 2013).

The GO and KEGG pathway annotations of the secretomes of M. abscessus ATCC 19977 and the MAB clinical isolates showed enrichment consistent with the characterization of previously reported mycobacterial secretomes (Cornejo-Granados et al., 2017; Gomez et al., 2015). Interestingly and in line with the increased secretome size as compared to MTB, the KEGG pathway analysis showed a high abundance of the Quorum sensing pathway for the predicted MAB secretomes, which was not present in our previous MTB secretome pathway analysis (Cornejo-Granados et al., 2017). The presence of a Quorum sensing pathway would be another similarity shared between MAB and non-mycobacterial pathogens commonly affecting patients with chronic lung disease such as Pseudomonas aeruginosa (Mukherjee and Bassler, 2019). In addition, it could be related to the ability of MAB to form biofilms (Clary et al., 2018; Orme and Ordway, 2014), further contributing to the capacity of MAB to tolerate antibiotics and to persist over long periods in the environment (Faria et al., 2015; Hunt-Serracin et al., 2019; Kulka et al., 2012; Maurer et al., 2014a).

The InterPro annotation showed that Mce domains were the most abundant $(2.14 \%)$ domains in the MAB reference secretome, while PPE and PE-PGRS domains only corresponded to $0.3 \%$ of the ES protein sequences. This tendency is contrary to our observations for MTB (Cornejo-Granados et al., 2017), where the PPE and PE-PGRS domains accounted for $\sim 12 \%$ of the secreted proteins and the Mce domains for only $0.5 \%$. The lower quantity of predicted PE/PPE proteins in MAB was somewhat expected. $M$. tuberculosis has five ESX secretion systems, four of which encode PE/PPE proteins, while MAB has only two (ESX-3 and ESX-4) of which only the ESX-3 operon includes PE/PPE genes (Dumas et al., 2016). In contrast, Mce domains are known for participating in host cell entry by mycobacteria (Kumar et al., 2005). Thus, their higher abundance in MAB as compared to MTB highlights the importance of this pathway for MAB survival within the host. It needs to be mentioned though that Kumar et al., 2005, also suggested that in low virulence bacteria, transport activities could be the primary function of Mce operons (Kumar et al., 2005). 
To compare the predicted secretomes according to colony morphotype, we first established the core secretome for the $\mathrm{R}$ and $\mathrm{S}$ variants per subspecies, thus eliminating individualities among the different isolates (Fig. 3). The high overall agreement between the core secretomes for both morphotypes of approximately $90 \%$ was expected, considering the fact that $\mathrm{R}$ variants can arise from the $\mathrm{S}$ morphotypes during persistent infection by loss of surface-exposed GPLs caused by mutations in the GPL synthesis pathway (Bernut et al., 2014; Catherinot et al., 2007; Roux et al., 2016; Ryan and Byrd, 2018). However, both the higher number and the higher antigenic densities (lower AAR values) of the ES proteins exclusively found in $\mathrm{R}$ variants indicate that additional genetic changes may evolve during S-to-R conversion. Moreover, this observation raises the question whether some strains with additional genetic traits associated with virulence are able to undergo $\mathrm{S}$-to-R conversion and cause disease due to $\mathrm{R}$ variants more easily than others. Genomic studies involving sequentially isolated $\mathrm{S}$ and $\mathrm{R}$ variants of the same strain obtained from individual patients over time will be required to better characterize the microevolution of MAB strains within the chronically infected host.

Similarly, the fact that MAB causes both chronic pulmonary disease (with $\mathrm{R}$ variants sometimes increasing over time) and extrapulmonary manifestations (mostly caused by $\mathrm{S}$ variants) led us to investigate whether differences exist in the predicted secretomes of isolates related to these clinical presentations. The absence of major differences in the GO, KEGG, and antigenicity analyses suggest that secretome variations do not influence MAB tissue tropism. Consequently, host characteristics such as severe immunosuppression may be the main driver for invasive MAB infections. Likewise, in the case of tissue infections, which often occur following surgical interventions, insufficient hygiene procedures and sterilization protocols for surgical equipment appear to be more relevant than pathobiological traits such as the secretome intrinsic to the causative MAB isolate (Maurer et al., 2014b)

Lastly, we observed that the predicted secretomes of all investigated clinical MAB isolates were less antigenic than the secretomes of M. tuberculosis H37Rv and two clinical M. tuberculosis isolates. Additionally, although there was no statistical difference, the isolates with a rough phenotype tended to be more antigenic that the isolates with smooth phenotype. Previous evidence with M. tuberculosis (Cornejo-Granados et al., 2017) showed that clinical isolates from the Beijing phenotype showed increased virulence and less antigenic secretomes than the reference strain H37Rv. Thus, the diminished antigenicity of MAB could be viewed as a virulence trait in itself as it would support colonization of the host for extended time periods without immediate progression into clinical disease. However, further experimental tests on antigenicity are needed to demonstrate this observation.

This study represents the first systematic prediction and in silico characterization of the MAB secretome. We acknowledge that an important constraint of this study is the limited total number of genomes analyzed per subspecies and biological source. Thus, care must be taken to not overinterpret the findings related to sample subcategories such as subspecies and morphotypes. Also, published experimental data on MAB secretomes are very limited and no systematic validation of the in silico findings reported herein could be performed against such datasets. Although, more research will be needed to determine experimental secretomes in NTM, our study demonstrates that using bioinformatics strategies can help to broadly explore mycobacterial secretomes including those of clinical isolates and to tailor subsequent, complex and time-consuming experimental approaches 
451 accordingly. This approach can support a systematic investigation of mycobacterial 452 secretomes exploring candidate proteins suitable for developing new vaccines and 453 diagnostic markers to distinguish between colonization and infection.

454

455

456

457

458

459

460

461

462

463

464

465

466

467

468

469

470

471

472

473

474

475

476

477

478

479

480

481

482

483

484

485

486

487

488

489

490

491

492

493

494

495

496

497 
499 Table 1| Clinical isolates metadata and number of ES proteins.

\begin{tabular}{|c|c|c|c|c|c|c|}
\hline Strain & Genome ID & Origin & Phenotype & \begin{tabular}{|c|} 
Total \\
predicted \\
proteins
\end{tabular} & $\begin{array}{c}\mathrm{ES} \\
\text { proteins }\end{array}$ & $\begin{array}{c}\% \mathrm{ES} \\
\text { proteins }\end{array}$ \\
\hline \multirow{7}{*}{$\begin{array}{c}\text { M. abscessus } \\
\text { subsp. abscessus }\end{array}$} & $4549-15$ & sputum & rough & 5,105 & 929 & 18 \\
\hline & $11351-15$ & sputum & rough & 5,138 & 966 & 19 \\
\hline & $8844-15$ & skin & smooth & 4,854 & 956 & 20 \\
\hline & $3563-15$ & sputum & smooth & 5,239 & 968 & 18 \\
\hline & $12389-15$ & sputum & smooth & 5,276 & 990 & 19 \\
\hline & $2677-16$ & sputum & smooth & 4,900 & 919 & 19 \\
\hline & $2572-17$ & $\begin{array}{c}\text { tissue (breast } \\
\text { implant) }\end{array}$ & NA & 4,847 & 874 & 18 \\
\hline \multirow{4}{*}{$\begin{array}{l}\text { M. abscessus } \\
\text { subsp. } \\
\text { massiliense }\end{array}$} & $14479-15$ & sputum & rough & 5,120 & 962 & 19 \\
\hline & 10896-16 & sputum & rough & 5,109 & 950 & 19 \\
\hline & $10003-15$ & sputum & smooth & 4,835 & 891 & 18 \\
\hline & $16155-15$ & sputum & smooth & 4,884 & 898 & 18 \\
\hline \multirow{4}{*}{$\begin{array}{l}\text { M. abscessus } \\
\text { subsp. bolletii }\end{array}$} & $11702-16$ & sputum & rough & 5,079 & 931 & 18 \\
\hline & $713-16$ & lymph node & rough & 5,456 & 1,037 & 19 \\
\hline & $7742-15$ & blood culture & smooth & 4,913 & 885 & 18 \\
\hline & $13116-16$ & lymph node & smooth & 5,305 & 990 & 19 \\
\hline $\begin{array}{c}\text { M. abscessus } \\
\text { subsp. abscessus }\end{array}$ & $\begin{array}{c}\text { reference } \\
\text { strain } \\
\text { ATCC19977 } \\
\text { (GenBank } \\
\text { CU458896.1) }\end{array}$ & - & - & 4,942 & 886 & 18 \\
\hline $\begin{array}{c}\text { M. tuberculosis } \\
\text { H37Rv }\end{array}$ & $\begin{array}{c}\text { reference } \\
\text { strain } \\
(\text { GenBank } \\
\text { AL123456.3) }\end{array}$ & - & - & 4,337 & 548 & 13 \\
\hline
\end{tabular}


Table 2. | Top 10 KEGG pathways assigned for M. abscessus ATCC19977 ES proteins.

\begin{tabular}{|c|l|c|}
\hline Ranking & \multicolumn{1}{|c|}{ Pathway name } & $\begin{array}{c}\text { Number of represented ES } \\
\text { proteins (\%) }\end{array}$ \\
\hline 1 & ABC transporters & $13(1.47)$ \\
\hline 2 & Two-component system & $9(1.02)$ \\
\hline 3 & Quorum sensing & $6(0.68)$ \\
\hline 4 & Oxidative phosphorylation & $4(0.45)$ \\
\hline 5 & Sulfur metabolism & $4(0.45)$ \\
\hline 6 & Glycerolipid metabolism & $4(0.45)$ \\
\hline 7 & Peptidoglycan biosynthesis & $4(0.45)$ \\
\hline 8 & Protein export & $4(0.45)$ \\
\hline 9 & Starch and sucrose metabolism & $3(0.34)$ \\
\hline 10 & Glyoxylate and dicarboxylate metabolism & $3(0.34)$ \\
\hline & & \\
\hline
\end{tabular}

Table 3| Top 10 most represented protein domains in M. abscessus ATCC19977 secretome.

\begin{tabular}{|c|c|c|}
\hline InterProcode & InterPro description & Number of ES proteins $(\%)$ \\
\hline IPR003399 & Mce/MlaD & $19(2.14)$ \\
\hline IPR026954 & PknH-like extracellular domain & $15(1.69)$ \\
\hline IPR032407 & Haemophore, haem-binding & $10(1.13)$ \\
\hline IPR020846 & Major facilitator superfamily domain & $7(0.79)$ \\
\hline IPR013766 & Thioredoxin domain & $6(0.68)$ \\
\hline IPR000064 & Endopeptidase, NLPC/P60 domain & $6(0.68)$ \\
\hline IPR001638 & $\begin{array}{c}\text { Solute-binding protein family 3/N- } \\
\text { terminal domain of MltF }\end{array}$ \\
\hline IPR000675 & Cutinase/acetylxylan esterase & $6(0.68)$ \\
\hline IPR005490 & L,D-transpeptidase catalytic domain & $5(0.56)$ \\
\hline IPR000073 & Alpha/beta hydrolase fold-1 & $5(0.56)$ \\
\hline
\end{tabular}


Table 4 | Abundance of Antigenic Regions (AAR) for M. abscessus and M. tuberculosis strains.

\begin{tabular}{|l|c|c|}
\hline \multicolumn{1}{|c|}{ Strain } & & $\begin{array}{c}\text { Average } \\
\text { AAR } \\
\text { value }\end{array}$ \\
\hline $\begin{array}{l}\text { M. tuberculosis Beijing isolate } \\
\text { C3* }\end{array}$ & Number of proteins in the dataset & \\
\hline $\begin{array}{l}\text { M. } \text { tuberculosis Beijing isolate } \\
\text { C4* }\end{array}$ & 553 & 37.52 \\
\hline M. bovis BCG Pasteur & 519 & 37.55 \\
\hline M. abscessus ATCC 19977 & 564 & 38.99 \\
\hline M. tuberculosis H37Rv & $\mathbf{8 8 6}$ & $\mathbf{4 0 . 7 8}$ \\
\hline M. abscessus clinical isolates & 548 & 40.63 \\
\hline & 680 & 41.54 \\
\hline
\end{tabular}

* both Beijing isolates were previously reported in Cornejo-Granados et al., 2017.

\section{Figure Legends}

Figure 1. GO enrichment analysis for the M. abscessus ATCC 19977 reference strain. Top 10 most enriched GO terms for the M. abscessus ATCC 19977 secretome (blue) and complete genome (red) in three categories: A) Cellular Component, B) Molecular Function and C) Biological Process.

Figure 2. Venn diagram between the core secretomes of the three M. abscessus subspecies. A) Number of total proteins contained in the core secretome of each subspecies. B) Shared and unique proteins between the three subspecies as per BLASTP (E-value 1.0E$3)$.

Figure 3. Venn diagram between the core secretomes of the three M. abscessus subspecies by colony morphotype. We used BLASTP (E-value 1.0E-3) to assess the core secretomes for isolates with rough and smooth colony morphotypes A) M. abscessus subsp. abscessus, B) M. abscessus subsp. massiliense and C) M. abscessus subsp. bolletii.

Figure 4. Comparison between AAR values for Excreted/Secreted (ES), non Excreted/Secreted (non-ES), intracellular (incell) and transmembrane (TM) proteins. AAR values were calculated for each of the 15 genomes sequenced. The $\mathrm{X}$-axis shows the cellular compartment and the Y-axis shows AAR values for the genomes of each subspecies: M. abscessus subsp. abscessus (green), M. abscessus subsp. bolletii (blue), M. abscessus subsp. massiliense (purple), M. abscessus ATCC19977 (red) and M. tuberculosis H37Rv (orange). Mann-Whitney tests were performed to compare the AAR of each group with a confidence level of $99 \%(* * *, p<0.001)$. 
Figure 5. Venn diagram between the predicted secretomes of $M$. tuberculosis $\mathbf{H 3 7 R v}$ and M. abscessus ATCC 19977. We used BLASTP (E-value 1.0E-3) to compare the complete secretomes of both species.

\section{Acknowledgements}

We would like to thank Julia Zallet and Vanessa Mohr for excellent technical assistance and Henrik Nielsen for his assistance with the SecrtomeP software. Parts of this work have been supported by the German Center for Infection Research and Grants by Joachim Herz Foundation, Hamburg, and Mukoviszidose Institut $\mathrm{gGmbH}$, Bonn, the research and development arm of the German Cystic Fibrosis Association Mukoviszidose e.V. to F.P.M. We acknowledge the support provided by CONACyT grant CB- 2013-223279 and SALUD-2014-C01-234188 to A.O.L. This research also received support by the DGAPA PAPIIT UNAM (IN215520) to A.O.L. F.C.G. acknowledges the support of CONACyT as a Postgraduate fellow.

\section{References}

Abeles, S. R., and Pride, D. T. (2014). Molecular Bases and Role of Viruses in the Human Microbiome. Journal of Molecular Biology 426, 3892-3906. doi:10.1016/j.jmb.2014.07.002.

Adekambi, T., Colson, P., and Drancourt, M. (2003). rpoB-based identification of nonpigmented and late-pigmenting rapidly growing mycobacteria. Journal of Clinical Microbiology 41, 5699-5708. doi:10.1128/jcm.41.12.5699-5708.2003.

Aziz, R. K., Bartels, D., Best, A. A., DeJongh, M., Disz, T., Edwards, R. A., et al. (2008). The RAST Server: rapid annotations using subsystems technology. BMC Genomics 9, 75. doi:10.1186/1471-2164-9-75.

Bachmann, N. L., Salamzade, R., Manson, A. L., Whittington, R., Sintchenko, V., Earl, A. M., et al. (2019). Key Transitions in the Evolution of Rapid and Slow Growing Mycobacteria Identified by Comparative Genomics. Front. Microbiol. 10, 3019. doi:10.3389/fmicb.2019.03019.

Bendtsen, J. D., Kiemer, L., Fausbøll, A., and Brunak, S. (2005a). Non-classical protein secretion in bacteria. BMC Microbiol 5, 58. doi:10.1186/1471-2180-5-58.

Bendtsen, J. D., Nielsen, H., Widdick, D., Palmer, T., and Brunak, S. (2005b). Prediction of twin-arginine signal peptides. BMC Bioinformatics 6, 167. doi:10.1186/1471-2105-6167.

Bernut, A., Herrmann, J.-L., Kissa, K., Dubremetz, J.-F., Gaillard, J.-L., Lutfalla, G., et al. (2014). Mycobacterium abscessus cording prevents phagocytosis and promotes abscess formation. Proc. Natl. Acad. Sci. U.S.A. 111, E943-52. doi:10.1073/pnas.1321390111.

Bolger, A. M., Lohse, M., and Usadel, B. (2014). Trimmomatic: a flexible trimmer for Illumina sequence data. Bioinformatics 30, 2114-2120. 
doi:10.1093/bioinformatics/btu170.

608

609

610

611

612

613

614

615

616

617

618

619

620

621

622

623

624

625

626

627

628

629

630

631

632

633

634

635

636

637

638

639

640

641

642

643

Catherinot, E., Clarissou, J., Etienne, G., Ripoll, F., Emile, J. F., Daffé, M., et al. (2007). Hypervirulence of a rough variant of the Mycobacterium abscessus type strain. Infection and Immunity 75, 1055-1058. doi:10.1128/IAI.00835-06.

Chen, J., Zhao, L., Mao, Y., Ye, M., Guo, Q., Zhang, Y., et al. (2019). Clinical Efficacy and Adverse Effects of Antibiotics Used to Treat Mycobacterium abscessus Pulmonary Disease. Front. Microbiol. 10, 1977. doi:10.3389/fmicb.2019.01977.

Clary, G., Sasindran, S. J., Nesbitt, N., Mason, L., Cole, S., Azad, A., et al. (2018). Mycobacterium abscessus Smooth and Rough Morphotypes Form AntimicrobialTolerant Biofilm Phenotypes but Are Killed by Acetic Acid. Antimicrobial Agents and Chemotherapy 62, 117. doi:10.1128/AAC.01782-17.

Conesa, A., and Götz, S. (2008). Blast2GO: A comprehensive suite for functional analysis in plant genomics. Int J Plant Genomics 2008, 619832. doi:10.1155/2008/619832.

Cornejo-Granados, F., Hurtado-Ramírez, J. M., Hernandez-Pando, R., and Ochoa-Leyva, A. (2018). Secret-AAR: a web server to assess the antigenic density of proteins and homology search against bacterial and parasite secretome proteins. Genomics. doi:10.1016/j.ygeno.2018.10.007.

Cornejo-Granados, F., Zatarain-Barrón, Z. L., Cantu-Robles, V. A., Mendoza-Vargas, A., Molina-Romero, C., Sánchez, F., et al. (2017). Secretome Prediction of Two M. tuberculosis Clinical Isolates Reveals Their High Antigenic Density and Potential Drug Targets. Front. Microbiol. 8, 128. doi:10.3389/fmicb.2017.00128.

Daugelat, S., Guile, H., Schoel, B., and Kaufmann, S. H. E. (1992). Secreted Antigens of Mycobacterium tuberculosis: Characterization with T Lymphocytes from Patients and Contacts after Two-Dimensional Separation. Journal of Infectious Diseases 166, 186190. doi:10.1093/infdis/166.1.186.

De Almeida, I. N., Da Silva Carvalho, W., Rossetti, M. L., Costa, E. R. D., and De Miranda, S. S. (2013). Evaluation of six different DNA extraction methods for detection of Mycobacterium tuberculosis by means of PCR-IS6110: preliminary study. BMC Research Notes 6, 561-6. doi:10.1186/1756-0500-6-561.

Dumas, E., Christina Boritsch, E., Vandenbogaert, M., Rodríguez de la Vega, R. C., Thiberge, J.-M., Caro, V., et al. (2016). Mycobacterial Pan-Genome Analysis Suggests Important Role of Plasmids in the Radiation of Type VII Secretion Systems. Genome Biol Evol 8, 387-402. doi:10.1093/gbe/evw001.

Faria, S., Joao, I., and Jordao, L. (2015). General Overview on Nontuberculous Mycobacteria, Biofilms, and Human Infection. J Pathog 2015, 809014-10. doi:10.1155/2015/809014.

Gomez, S., Adalid-Peralta, L., Palafox-Fonseca, H., Cantu-Robles, V. A., Soberón, X., 
644

Sciutto, E., et al. (2015). Genome analysis of Excretory/Secretory proteins in Taenia solium reveals their Abundance of Antigenic Regions (AAR). Sci. Rep. 5, 9683. doi:10.1038/srep09683.

Gupta, M. K., Subramanian, V., and Yadav, J. S. (2009). Immunoproteomic identification of secretory and subcellular protein antigens and functional evaluation of the secretome fraction of Mycobacterium immunogenum, a newly recognized species of the Mycobacterium chelonae-Mycobacterium abscessus group. J. Proteome Res. 8, 23192330. doi:10.1021/pr8009462.

Gutiérrez, A. V., Viljoen, A., Ghigo, E., Herrmann, J.-L., and Kremer, L. (2018). Glycopeptidolipids, a Double-Edged Sword of the Mycobacterium abscessus Complex. Front. Microbiol. 9, 1145. doi:10.3389/fmicb.2018.01145.

Howard, S. T., Rhoades, E., Recht, J., Pang, X., Alsup, A., Kolter, R., et al. (2006). Spontaneous reversion of Mycobacterium abscessus from a smooth to a rough morphotype is associated with reduced expression of glycopeptidolipid and reacquisition of an invasive phenotype. Microbiology 152, 1581-1590. doi:10.1099/mic.0.28625-0.

Hunt-Serracin, A. C., Parks, B. J., Boll, J., and Boutte, C. C. (2019). Mycobacterium abscessus Cells Have Altered Antibiotic Tolerance and Surface Glycolipids in Artificial Cystic Fibrosis Sputum Medium. Antimicrobial Agents and Chemotherapy 63, 1370. doi:10.1128/AAC.02488-18.

Jarand, J., Levin, A., Zhang, L., Huitt, G., Mitchell, J. D., and Daley, C. L. (2011). Clinical and microbiologic outcomes in patients receiving treatment for Mycobacterium abscessus pulmonary disease. Clin. Infect. Dis. 52, 565-571. doi:10.1093/cid/ciq237.

Juncker, A. S., Willenbrock, H., Heijne, von, G., Brunak, S., Nielsen, H., and Krogh, A. (2003). Prediction of lipoprotein signal peptides in Gram-negative bacteria. Protein Sci. 12, 1652-1662. doi:10.1110/ps.0303703.

Käll, L., Krogh, A., and Sonnhammer, E. L. L. (2007). Advantages of combined transmembrane topology and signal peptide prediction--the Phobius web server. Nucleic Acids Research 35, W429-32. doi:10.1093/nar/gkm256.

Kulka, K., Hatfull, G., and Ojha, A. K. (2012). Growth of Mycobacterium tuberculosis biofilms. JoVE. doi:10.3791/3820.

Kumar, A., Chandolia, A., Chaudhry, U., Brahmachari, V., and Bose, M. (2005). Comparison of mammalian cell entry operons of mycobacteria: in silico analysis and expression profiling. FEMS Immunol. Med. Microbiol. 43, 185-195. doi:10.1016/j.femsim.2004.08.013.

Laencina, L., Dubois, V., Le Moigne, V., Viljoen, A., Majlessi, L., Pritchard, J., et al. (2018). Identification of genes required for Mycobacterium abscessus growth in vivo with a prominent role of the ESX-4 locus. Proc. Natl. Acad. Sci. U.S.A. 115, E1002- 
Lee, M.-R., Sheng, W.-H., Hung, C.-C., Yu, C.-J., Lee, L.-N., and Hsueh, P.-R. (2015). Mycobacterium abscessus Complex Infections in Humans. Emerg. Infect. Dis. 21, 1638-1646. doi:10.3201/2109.141634.

686

687

688
Malhotra, S., Vedithi, S. C., and Blundell, T. L. (2017). Decoding the similarities and differences among mycobacterial species. PLoS Negl Trop Dis 11, e0005883. doi:10.1371/journal.pntd.0005883.

Maurer, F. P., Bruderer, V. L., Ritter, C., Castelberg, C., Bloemberg, G. V., and Böttger, E. C. (2014a). Lack of antimicrobial bactericidal activity in Mycobacterium abscessus. Antimicrobial Agents and Chemotherapy 58, 3828-3836. doi:10.1128/AAC.02448-14.

Maurer, F., Castelberg, C., Braun, von, A., Wolfensberger, A., Bloemberg, G., Bottger, E., et al. (2014b). Postsurgical wound infections due to rapidly growing mycobacteria in Swiss medical tourists following cosmetic surgery in Latin America between 2012 and 2014. Euro Surveill. 19, 20905. doi:10.2807/1560-7917.es2014.19.37.20905.

Moriya, Y., Itoh, M., Okuda, S., Yoshizawa, A. C., and Kanehisa, M. (2007). KAAS: an automatic genome annotation and pathway reconstruction server. Nucleic Acids Research 35, W182-5. doi:10.1093/nar/gkm321.

Mukherjee, S., and Bassler, B. L. (2019). Bacterial quorum sensing in complex and dynamically changing environments. Nature Publishing Group 17, 371-382. doi:10.1038/s41579-019-0186-5.

Nessar, R., Cambau, E., Reyrat, J.-M., Murray, A., and Gicquel, B. (2012). Mycobacterium abscessus: a new antibiotic nightmare. J. Antimicrob. Chemother. 67, 810-818. doi:10.1093/jac/dkr578.

Nurk, S., Bankevich, A., Antipov, D., Gurevich, A. A., Korobeynikov, A., Lapidus, A., et al. (2013). Assembling single-cell genomes and mini-metagenomes from chimeric MDA products. J. Comput. Biol. 20, 714-737. doi:10.1089/cmb.2013.0084.

O'Toole, N., Min, X. J., Butler, G., Storms, R., and Tsang, A. (2013). Sequence-Based Analysis of Fungal Secretomes. Elsevier B.V doi:10.1016/S1874-5334(06)80015-8.

Orme, I. M., and Ordway, D. J. (2014). Host response to nontuberculous mycobacterial infections of current clinical importance. Infection and Immunity 82, 3516-3522. doi:10.1128/IAI.01606-13.

Petersen, T. N., Brunak, S., Heijne, von, G., and Nielsen, H. (2011). SignalP 4.0: discriminating signal peptides from transmembrane regions. Nature Publishing Group 8, 785-786. doi:10.1038/nmeth.1701.

Ripoll, F., Deshayes, C., Pasek, S., Laval, F., Beretti, J.-L., Biet, F., et al. (2007). Genomics of glycopeptidolipid biosynthesis in Mycobacterium abscessus and M. chelonae. BMC 
Genomics 8, 114-9. doi:10.1186/1471-2164-8-114.

Ripoll, F., Pasek, S., Schenowitz, C., Dossat, C., Barbe, V., Rottman, M., et al. (2009). Non mycobacterial virulence genes in the genome of the emerging pathogen Mycobacterium abscessus. PLoS ONE 4, e5660. doi:10.1371/journal.pone.0005660.

Roux, A.-L., Viljoen, A., Bah, A., Simeone, R., Bernut, A., Laencina, L., et al. (2016). The distinct fate of smooth and rough Mycobacterium abscessus variants inside macrophages. Open Biol 6, 160185. doi:10.1098/rsob.160185.

Ryan, K., and Byrd, T. F. (2018). Mycobacterium abscessus: Shapeshifter of the Mycobacterial World. Front. Microbiol. 9, 2642. doi:10.3389/fmicb.2018.02642.

Sanguinetti, M., Ardito, F., Fiscarelli, E., La Sorda, M., D'Argenio, P., Ricciotti, G., et al. (2001). Fatal pulmonary infection due to multidrug-resistant Mycobacterium abscessus in a patient with cystic fibrosis. Journal of Clinical Microbiology 39, 816-819. doi:10.1128/JCM.39.2.816-819.2001.

Shin, A.-R., Sohn, H., Won, C. J., Lee, B., Kim, W. S., Kang, H. B., et al. (2010). Characterization and identification of distinct Mycobacterium massiliense extracellular proteins from those of Mycobacterium abscessus. J. Microbiol. 48, 502-511. doi:10.1007/s12275-010-0038-5.

Sonnhammer, E. L., Heijne, von, G., and Krogh, A. (1998). A hidden Markov model for predicting transmembrane helices in protein sequences. Proc Int Conf Intell Syst Mol Biol 6, 175-182.

Tjalsma, H., Antelmann, H., Jongbloed, J. D. H., Braun, P. G., Darmon, E., Dorenbos, R., et al. (2004). Proteomics of protein secretion by Bacillus subtilis: separating the "secrets" of the secretome. Microbiol. Mol. Biol. Rev. 68, 207-233. doi:10.1128/MMBR.68.2.207-233.2004.

Tortoli, E., Fedrizzi, T., Meehan, C. J., Trovato, A., Grottola, A., Giacobazzi, E., et al. (2017). The new phylogeny of the genus Mycobacterium: The old and the news. Infect. Genet. Evol. 56, 19-25. doi:10.1016/j.meegid.2017.10.013.

Tortoli, E., Kohl, T. A., Brown-Elliott, B. A., Trovato, A., Leão, S. C., Garcia, M. J., et al. (2016). Emended description of Mycobacterium abscessus, Mycobacterium abscessus subsp. abscessus and Mycobacteriumabscessus subsp. bolletii and designation of Mycobacteriumabscessus subsp. massiliense comb. nov. Int. J. Syst. Evol. Microbiol. 66, 4471-4479. doi:10.1099/ijsem.0.001376.

Waman, V. P., Vedithi, S. C., Thomas, S. E., Bannerman, B. P., Munir, A., Skwark, M. J., et al. (2019). Mycobacterial genomics and structural bioinformatics: opportunities and challenges in drug discovery. Emerg Microbes Infect 8, 109-118. doi:10.1080/22221751.2018.1561158.

Wang, S., Wei, W., and Cai, X. (2015). Genome-wide analysis of excretory/secretory 
755

756

757

758

759

760

761

762

763

764

765

766

767

768

769

770

771

772

773

774

775

776

777

778

779

780

781

782

proteins in Echinococcus multilocularis: insights into functional characteristics of the tapeworm secretome. Parasites \& Vectors 8, 666. doi:10.1186/s13071-015-1282-7.

Yadav, J. S., and Gupta, M. (2012). Secretome differences between the taxonomically related but clinically differing mycobacterial species Mycobacterium abscessus and $\mathrm{M}$. chelonae. JIOMICS 2, 1-16. doi:10.5584/jiomics.v2i2.98.

Zdobnov, E. M., and Apweiler, R. (2001). InterProScan - an integration platform for the signature-recognition methods in InterPro. Bioinformatics 17, 847-848. doi:10.1093/bioinformatics/17.9.847.

Zheng, J., Ren, X., Wei, C., Yang, J., Hu, Y., Liu, L., et al. (2013). Analysis of the secretome and identification of novel constituents from culture filtrate of bacillus Calmette-Guerin using high-resolution mass spectrometry. Mol. Cell Proteomics 12, 2081-2095. doi:10.1074/mcp.M113.027318.

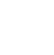




\section{$784 \quad$ Figure 1.}

\section{A) Cellular Component}

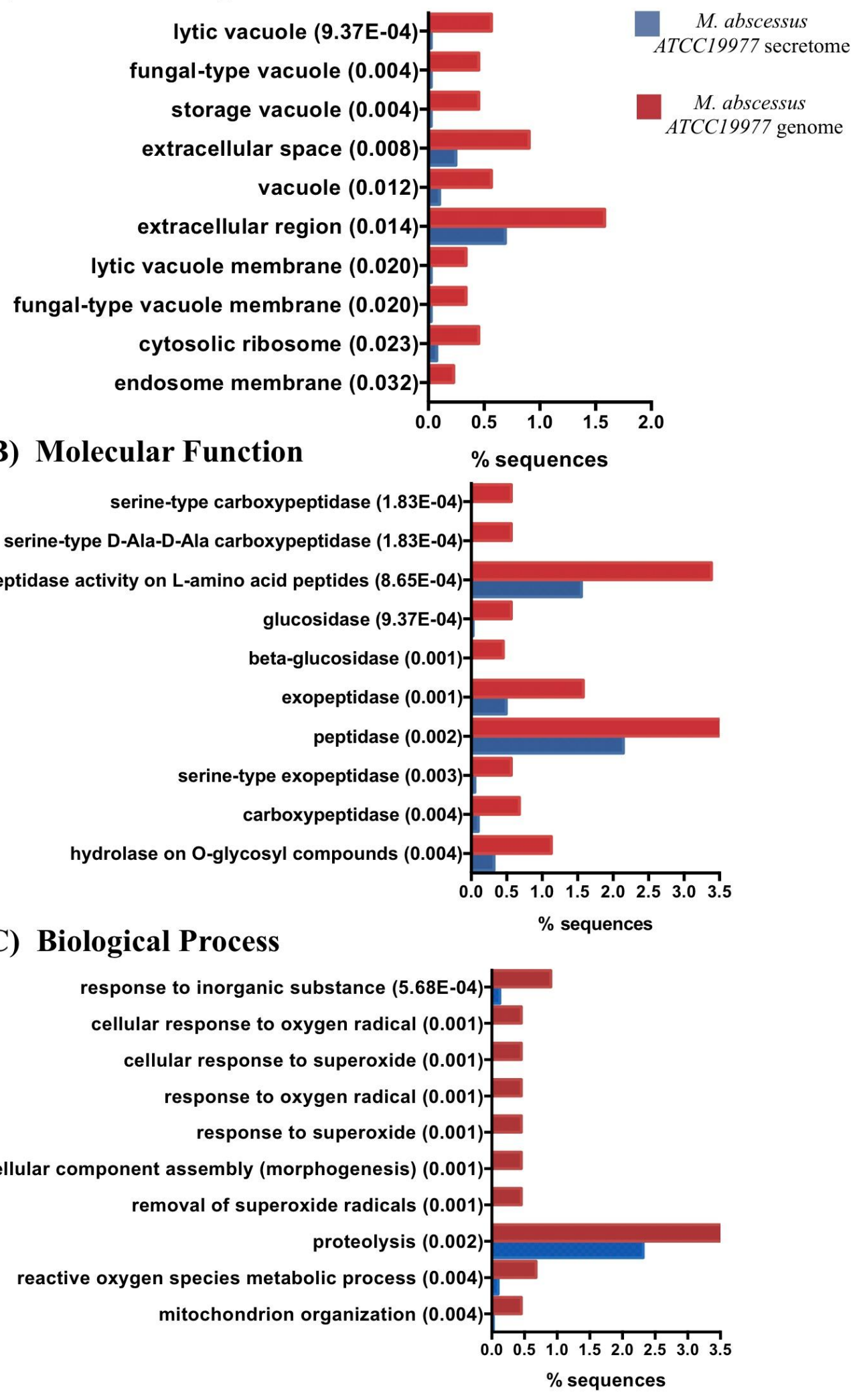


A)

7 individual secretomes of M. abs sbsp. abscessus isolates

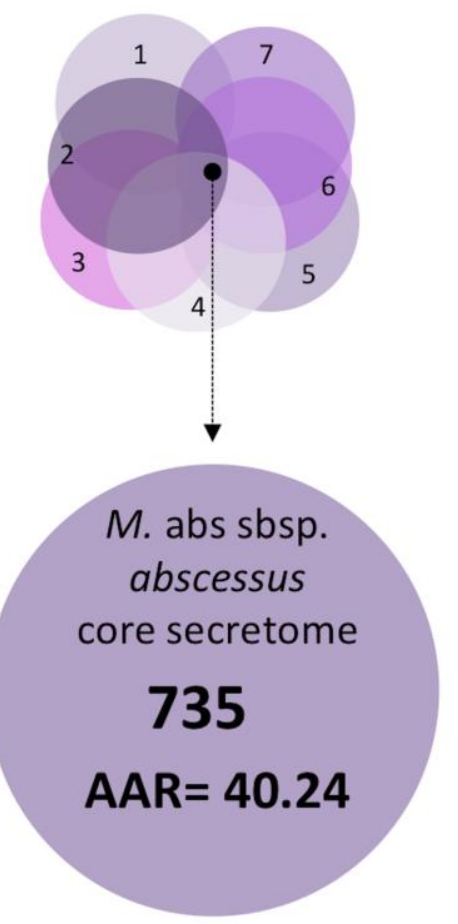

4 individual secretomes of M. abs sbsp. massiliense isolates

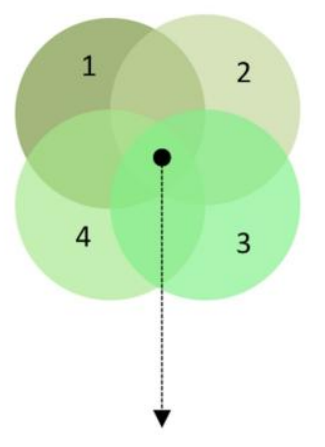

\section{M. abs} subsp.massiliense core secretome

813

\section{$A A R=41.38$}

4 individual secretomes of M. abs sbsp. bolletii isolates

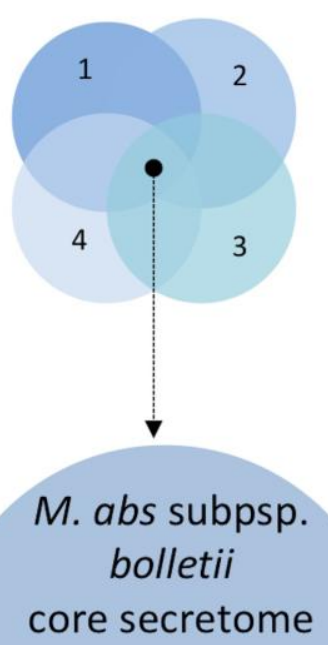

794

\section{AAR $=40.75$}

B)

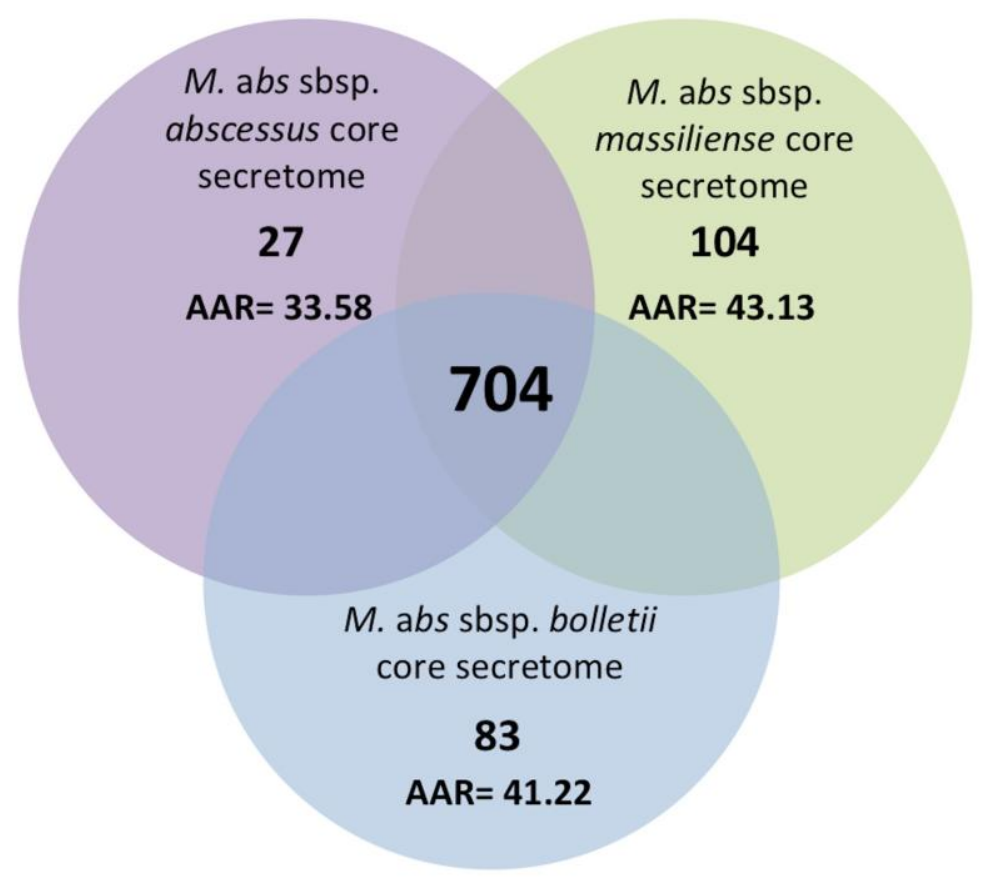


bioRxiv preprint doi: https://doi.org/10.1101/2020.10.22.349720; this version posted October 22, 2020. The copyright holder for this preprint (which was not certified by peer review) is the author/funder. All rights reserved. No reuse allowed without permission.

\section{$788 \quad$ Figure 3.}




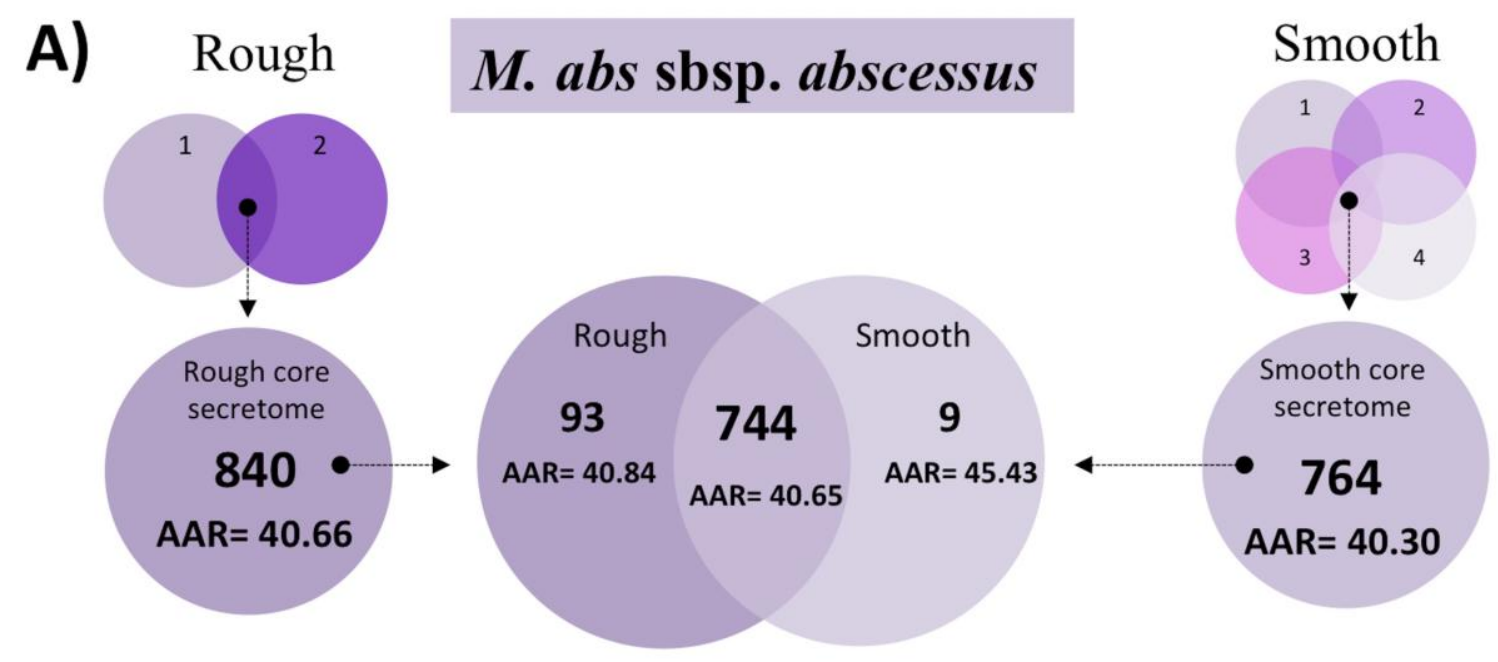

B) Rough

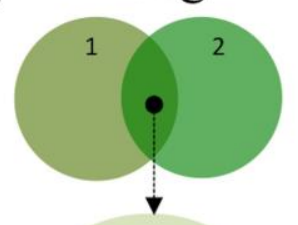

Rough core secretome

924

AAR $=\mathbf{4 0 . 8 5}$

\section{M. abs sbsp. massiliense}

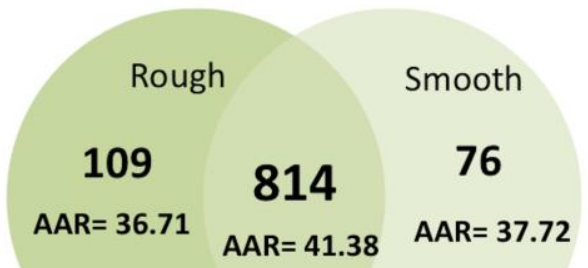

\section{M. abs sbsp. bolleti}
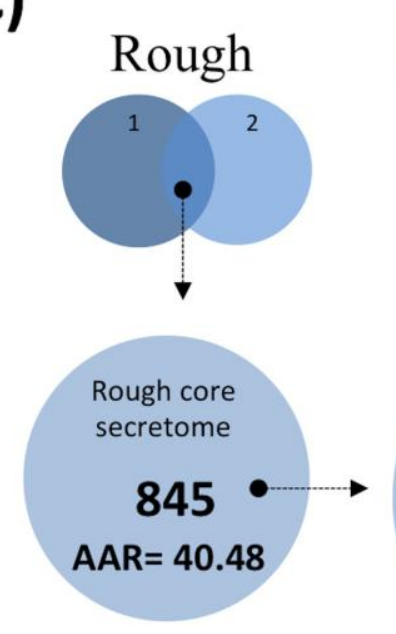

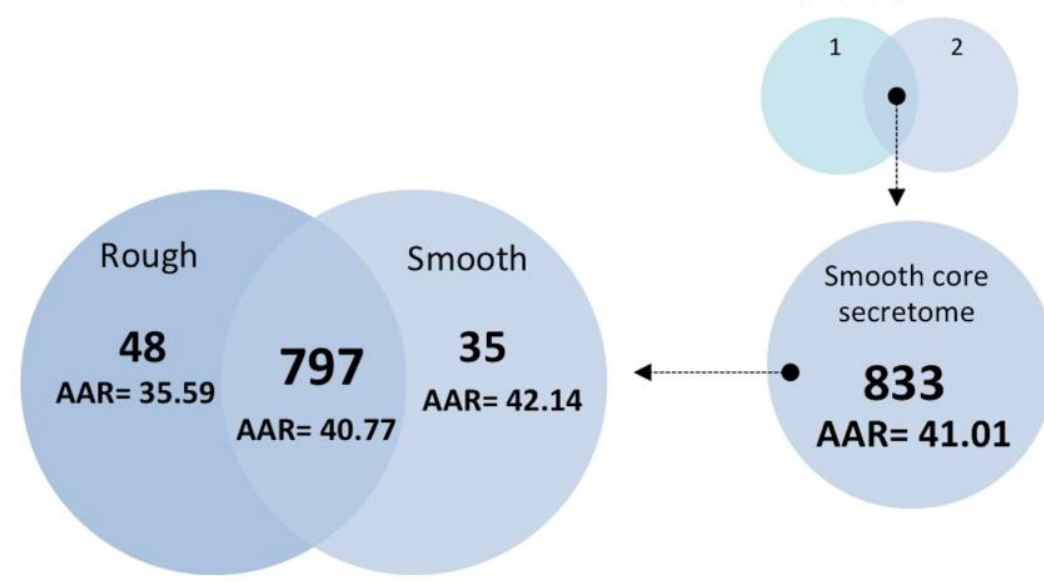

Smooth

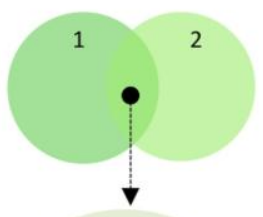

Smooth core secretome

872

AAR $=41.08$

C) 
bioRxiv preprint doi: https://doi.org/10.1101/2020.10.22.349720; this version posted October 22, 2020. The copyright holder for this preprint (which was not certified by peer review) is the author/funder. All rights reserved. No reuse allowed without permission.

\section{$790 \quad$ Figure 4.}

791

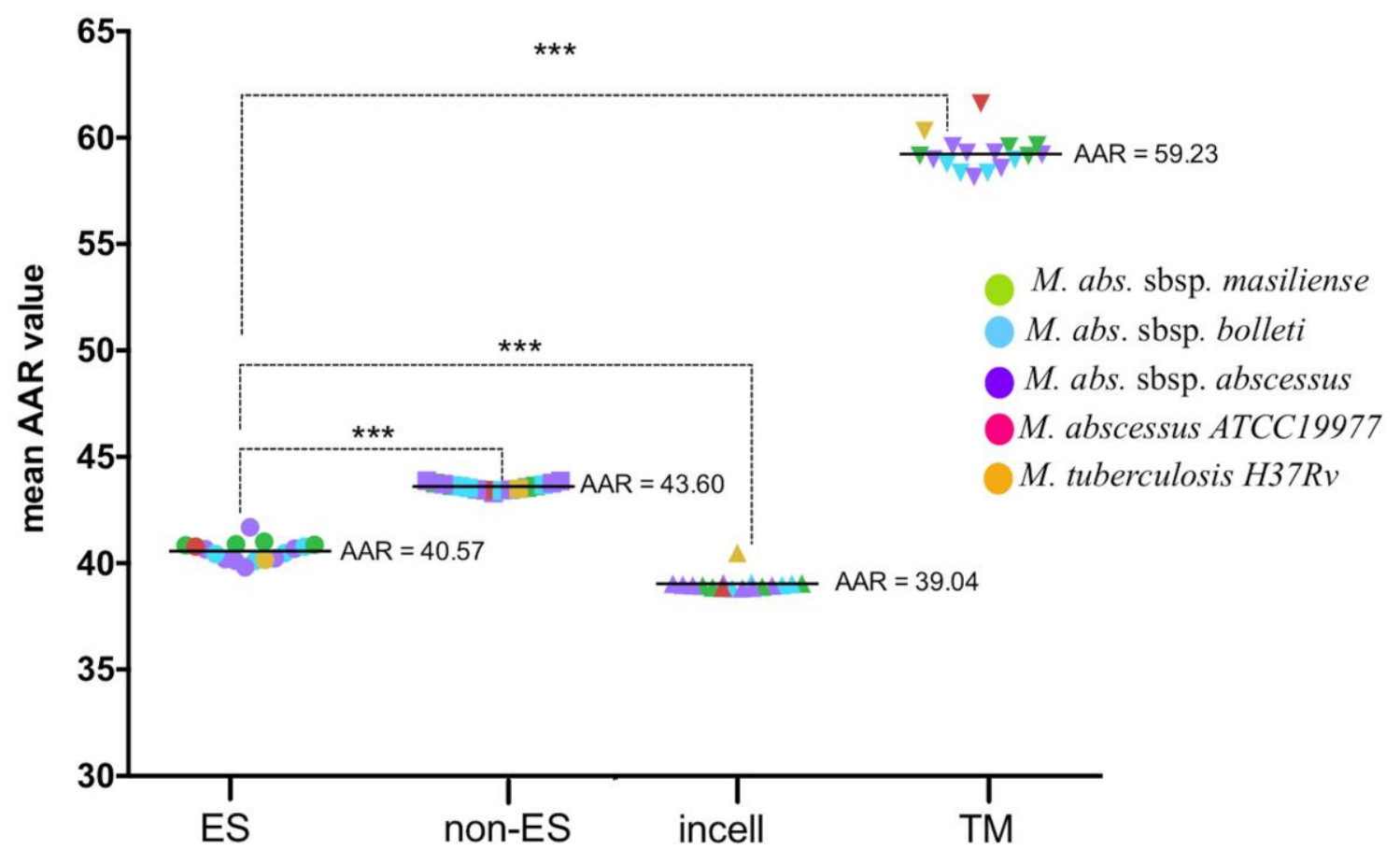

792

793

794

795

796

797

798

799

800

801

802

803

804 
Figure 5.

\section{M.tuberculosis \\ H37Rv secretome}

548

$A A R=40.37$
M. abscessus

\section{ATCC19977}

secretome

886

$A A R=39.63$
M.tuberculosis

\section{3}

$A A R=35.24$
M. abscessus

\section{7}

$A A R=38.88$

623

$A A R=40.07$

806 